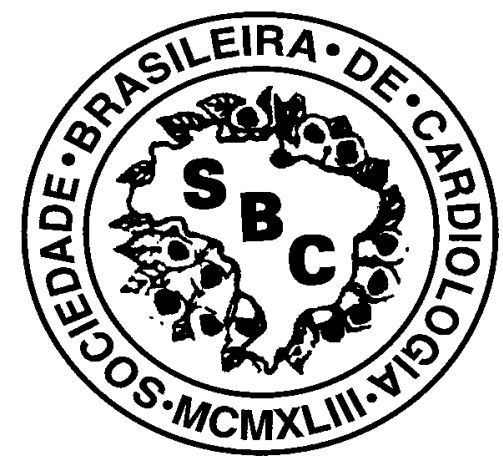

Diretriz para Realização de Exames Diagnósticos e Terapêuticos em Hemodinâmica 


\title{
Diretriz para Realização de Exames Diagnósticos e Terapêuticos em Hemodinâmica
}

\author{
Editor \\ Jamil Abdalla Saad \\ Editor Associado \\ José Carlos de Faria Garcia \\ Coordenação \\ Jorge Ilha Guimarães
}

\section{Introdução}

Este documento tem o objetivo de estabelecer as normas essenciais para que os Serviços de Hemodinâmica,em nosso país, estejam aptos a realizar, de maneira segura e eficaz, procedimentos diagnósticos e terapêuticos em Cardiologia Intervencionista.

Torna-se, do nosso ponto de vista, necessária e oportuna a realização de um trabalho desta natureza, uma vez que, por não dispormos desta opção até o momento, ficamos sujeitos à importação indiscriminada de normas e consensos extraídos de outras realidades sócio-econômicas e culturais, gerando problemas, não só pela tentativa de reproduzir inapropriadamente hábitos não condizentes com nossa prática e recursos, mas também por uma eventual negligência com exigências indispensáveis, que por não estarem facilmente disponíveis em nossa rotina, correm o risco de serem julgadas dispensáveis, já que advindas de outras realidades.

Deve-se ainda ressaltar a importância deste trabalho como tentativa de acompanhare corresponder ao extraordinário avanço que a especialidade experimentou nos últimos 10 anos Esse fato acrescentou significativa complexidade, mesmo às atividades corriqueiras, além de ampliar, sobremaneira, as indicações das técnicas, não só para as cardiopatias, mas também para o território vascular extracardíaco, área em que o cardiologista intervencionista vem atuando de maneira crescente.

Por outro lado, a pressão pela redução de custos exigidos nos últimos anos, não pode chegar, dada a ausência de normas ou fiscalizações rotineiras, a comprometero resultadoe colocar em risco intervenções sofisticadas e reconhecidamente eficazes no tratamento das doenças cardiovasculares.

Serão abordadas neste documento as exigências de ordem geral para a prática da Cardiologia Intervencionista, contendo em outros documentos as diretrizes específicas para a indicação e acompanhamento das intervenções diagnósticas e terapêuticas.

\section{Instalações do laboratório de hemodinâmica}

As instalações necessárias para a realização de procedimentos diagnósticos e terapêuticos em Cardiologia Intervencionista assemelham-se às necessárias para o treinamento de médicos nessa área.
O rigor com o equipamento básico, desfibriladores, oxímetros, monitores, acessórios de anestesia e todo material para atendimento a urgências médicas deve ser tal que permita o rápido e eficiente atendimento a pacientes em estado crítico.

Obrigatoriamente, o serviço deve possuir, pelo menos, um equipamento dotado de imagem digital de alta qualidade, com aquisição em tempo real (matrix 512 X 512 X 8 - bites a 30 quadros por segundo), condição essencial para o julgamento diagnóstico adequado, bem como para a otimização do resultado da intervenção. $O$ registro e o arquivamento dos procedimentos devem, preferencialmente, ser em formato digital, embora essa não seja uma norma obrigatória, pois alguns serviços ainda utilizam filme $35 \mathrm{~mm}$. Polígrafo de no mínimo, 3 canais, permitindo registro simultâneo de 2 pressões e do eletrocardiograma, também é considerado item obrigatório.

É fundamental que o laboratório possua aparelho para medida do tempo de coagulação ativado (TCA) na sala de hemodinâmica.

Balão intra-aórtico, apesar de ser item obrigatório no hospital, não precisa estar disponível em caráter dedicado ao laboratório de hemodinâmica. Também não são itens obrigatórios o ultra-som intracoronariano e os equipamentos que permitem o estudo funcional das artérias coronárias.

Controle de qualidade de imagem deve ser realizado periodicamente (com análise pormenorizada, pelo menos, 2 vezes ao ano), assim como o controle da emissão de radiografia, tanto pela segurança da equipe operacional, como pela dos freqüentadores ocasionais do ambiente e dos pacientes examinados. É obrigatório o controle mensal da exposição à radiografia de toda a equipe funcional através da avaliação de dosímetros, sob coordenação da Comissão Nacional de Energia Nuclear (CNEN), conforme normas internacionalmente estabelecidas.

Um aspecto relevante, que tem adquirido importância crescente, refere-se à transferência de dados entre os serviços, sendo obrigatório o envio de imagens gravadas em filme de $35 \mathrm{~mm}$ ou digitalizadas em padrão DICOM, não devendo ser aceitas, para definição e conduta, imagens gravadas em fitas para aparelhos de vídeo-cassete.

Por fim, o laboratório de hemodinâmica deve manter registro de todos os seus casos, pelo período mínimo de 5 anos, preferentemente na forma digital, incluindo exames 
diagnósticos e terapêuticos, a fim de permitir a recuperação de dados quanto ao crédito dos exames e, especialmente, quanto a intercorrências e resultados de procedimentos. Este banco de dados é necessário para contínuo monitoramento de qualidade no laboratório, permitindo ainda o acompanhamento ou revisão do registro de casos pela entidade de classe, evitando-se fraudes no sistema de relatos.

\section{Instalações do hospital}

Conforme estabelecido na portaria $227 \mathrm{de} 05$ de abril de 2002 da Secretaria de Assistência à Saúde do Ministério da Saúde (SAS - MS), o atendimento em Cardiologia Intervencionista deve ser realizado em Centros de Referência em Assistência Cardiovascular de Alta Complexidade, com atendimento global ao cardiopata, contendo as características a seguir enumeradas para seu funcionamento:

3.1 - Materiais e equipamentos - O Centro deve possuir todos os materiais/equipamentos necessários, em perfeito estado de conservação e funcionamento, para assegurar a qualidade da assistência aos pacientes e para possibilitar o diagnóstico, tratamento/acompanhamento médico e de enfermagem, fisioterápico,nutricional e dietético. Além dos materiais/equipamentos gerais do hospital e da a existência de enfermarias para internação clínica e cirúrgica, o Centro deve possuir os seguintes materiais e equipamentos mínimos:

O Serviço de Cirurgia Cardiovascular deve contar com Centro Cirúrgico equipado com: colchão térmico; circulação extra-corpórea; desfibrilador com pás externas e internas; marca-passo externo provisório; balão intra-aórtico; monitor de pressão invasiva, portátil ou modular; oxímetro de pulso; 2 termômetros; 4 bombas de infusão, no mínimo; aparelho para controle de coagulação por TCA.

3.2 - Recursos diagnósticos e terapêuticos - Além dos já enunciados, o Centro deve contar com os seguintes recursos diagnósticos e terapêuticos: Laboratório de Análises Clínicas, disponível nas 24 h do dia, onde se realizem exames de bioquímica; hematologia; microbiologia, gasometria e líquidos orgânicos, inclusive líquor e dosagens enzimáticas específicas. O Laboratório deve possuir Certificado de Controle de Qualidade; Unidade de Imagem: aparelho de radiografia, RX portátil, ultra-sonografia, ecodopplercardiografia transtorácica; Hemoterapia disponível nas 24h do dia; Unidade de Tratamento Intensivo, equivalente às cadastradas pelo SUS e classificada como de Tipo II ou III ou Especializada, de acordo com a Portaria GM/MS nº 3432, de 12 de agosto de 1998

3.3 - Funcionamento integrado-Embora, na atualidade, não se conceba mais o funcionamento do Centro de Cardiologia Intervencionista sem a possibilidade de realização de procedimentos terapêuticos, poderá a unidade, em caráter provisório e pelo período de seis meses, realizar cateterismos diagnósticos, enquanto aguarda a complementação da estrutura hospitalar em sua referida complexidade, mais especificamente quanto à necessidade de possuir Serviço de Cirurgia Cardíaca. Nesses casos, excluem-se os pacientes nas condições abaixo, conforme estabelecido em outras publicações: idade > 75 anos; ICC classe III ou IV (NYHA); síndrome coronariana aguda de risco intermediário / alto; isquemia pós -
IAM; edema pulmonar de provável etiologia isquêmica; testes isquêmicos sugestivos de coronariopatia multivascular ou lesão de tronco de coronária esquerda; lesão de tronco de coronária esquerda; grave disfunção valvar associada à disfunção ventricular; risco acentuado de complicações vasculares; doença congênita complexa em adulto; pacientes pediátricos de maneira ampla; procedimentos terapêuticos percutâneos de maneira geral.

A necessidade, dentro da estrutura hospitalar, de centro cirúrgico e de equipe habilitada em cirurgia cardíaca para a prática da Cardiologia Intervencionista tem gerado dúvidas e, por vezes, conflitos quanto ao real papel que o cirurgião cardiovascular exerce quando na condição de suporte ao cardiologista intervencionista. Deve ser entendido que essa situação não demanda a paralisação do bloco cirúrgico e do trabalho do cirurgião, enquanto se realizam intervenções percutâneas terapêuticas pelo cardiologista intervencionista. O cirurgião deverá estar ciente da realização do procedimento, porém dedicado ao seu trabalho rotineiro. $\mathrm{O}$ advento de tecnologias que permitem controlar e, freqüentemente, tratar as complicações do cateterismo cardíaco diagnóstico e terapêutico reduziu, sensivelmente, a necessidade de encaminhar o paciente ao bloco cirúrgico para este fim. Por outro lado, caso a disponibilidade exigida do cirurgião cardiovascular fosse integral, com paralisação de suas atividades e do bloco cirúrgico, as intervenções cardíacas percutâneas só poderiam ser realizadas no interior do bloco cirúrgico, para uma abordagem emergencial das complicações, o que não se aplica a nenhum país do mundo.

\section{Responsável técnico}

O responsável técnico pelo Serviço de Hemodinâmica deverá ser membro titular da Sociedade Brasileira de Hemodinâmica e Cardiologia Intervencionista (SBHCI), com título de habilitação em Hemodinâmica (área de atuação), conferido pela AMB em convênio com a Sociedade Brasileira de Cardiologia (SBC).

\section{Seleção do Contraste iodado}

Todos os contrastes modernos em utilização nos dias atuais são à base de iodo orgânico, o qual, devido ao seu número atômico elevado e versatilidade química, provou ser de excelente aplicabilidade para opacificação intravascular.

5.1 - Classificação - a) Alta osmolaridade - contrastes iônicos: osmolaridade variável, porém 5-8 x osmolaridade plasmática. Exibem ph entre 6,0 e 7,0, concentração de sódio 1-7x maior que a plasmática e contêm aditivos que quelam o cálcio iônico. Possuem propriedades antitrombóticas expressivas; b) Baixa osmolaridade - contrastes iôni$\cos \rightarrow$ Redução da osmolaridade superior a $60 \%$, com conseqüente redução dos efeitos relacionados à hipertonicidade. Não quelam o cálcio iônico e mantêm as propriedades antitrombóticas; Não iônicos $\rightarrow$ redução da osmolaridade superior a 50\%. Não quelam o cálcio iônico e possuem propriedades antitrombóticas reduzidas; c) Isosmolares: osmolaridade semelhante à plasmática, com significativa redução dos efeitos atribuídos à hiperosmolaridade. 


\section{2 - Contrastes disponíveis}

\begin{tabular}{|lcccc|}
\hline \multicolumn{1}{|c}{ Classe } & Nome genérico & Osmolaridade (mOsm/kg-H20) & Efeito quelante $C a$ & Efeito antitrombótico \\
\hline Iônico - Alta osmolaridade & Diatrizoato & 2076 & $(+)$ & $(+)$ \\
Iônico - Alta osmolaridade & Metrizoato & 1797 & $(+)$ & $(++)$ \\
Iônico - Alta osmolaridade & Iotalmato & 1797 & $(+)$ & $(++)$ \\
Iônico - Baixa osmolaridade & Ioxaglato & 600 & $(-)$ & $(++)$ \\
Não iônico - Baixa osmolaridade & Iopamidol & 796 & $(-)$ & $(+)$ \\
Não iônico - Baixa osmolaridade & Iohexol & 844 & $(-)$ & $(+)$ \\
Não iônico - Baixa osmolaridade & Ioversol & 792 & $(-)$ & \# \\
Não iônico - Baixa osmolaridade & Ioxilan & 695 & $(-)$ & \\
Não iônico - Isosmolar & Iodixanol & 290 & & \\
\hline \# Dado comparativo não disponível. & & & \\
* Osmolaridade sérica normal: 275-295 mOsmol/Kg-H2O. & & & \\
\hline
\end{tabular}

5.3 - Efeitos colaterais - Os sintomas mais comuns descritos após procedimentos angiográficos são urticária, prurido, náusea, vômito e sensação de calor, todos apresentando evolução favorável e ocorrendo com menor freqüência com a utilização de contrastes de baixa osmolaridade. Outros efeitos colaterais de menor incidência, mas de importância clínica maior, são: a) efeitos eletrofisiológicos - a injeção intracoronariana ou intracardíaca de contrastes iodados produz efeitos eletrofisiológicos significativos. Há redução da freqüência de despolarização do nó sinusal com conseqüente bradicardia transitória e até parada sinusal. Ocorre alentecimento da condução ao nível do nó $\mathrm{AV}$, com aumento do intervalo Pr, e possível ocorrência de bloqueios atrioventriculares avançados, efeitos explicados pelas propriedades quelantes de cálcio desses contrastes. Também há a ocorrência de alterações na configuração da onda $\mathrm{T}$ de significado desconhecido. A fibrilação ventricular ocorre em, aproximadamente, 1 em cada 200 procedimentos angiográficos e é explicada, em parte, também pela hipocalcemia transitória. A incidência de efeitos eletrofisiológicos adversos é menor com as formulações de baixa osmolaridade, no entanto não há recomendação explícita, baseada em evidência científica, de que essa diminuição reduza os custos hospitalares ou melhore os resultados clínicos; b) efeitos hemodinâmicos: são constituídos pela vasodilatação arterial sistêmica (mais evidente após a ventriculografia) e a depressão da contratilidade miocárdica, que estão relacionadas as propriedades quelantes de cálcio iônico. A depressão da contratilidade e a conseqüente hipotensão são seguidas por 8-10s de hipertensão rebote compensatória e podem exacerbar isquemia miocárdica, às vezes refratária, em pacientes mais susceptíveis. Esses efeitos são menos freqüentes com a utilização de agentes de baixa osmolaridade; c) nefrotoxicidade: a administração de contrastes iodados pode provocar a ocorrência de insuficiência renal aguda, geralmente não oligúrica, ocorrendo, habitualmente, com a elevação transitória das provas de função renal (com pico em 3-5 dias), às vezes com necessidade de tratamento dialítico e até progressão para insuficiência renal crônica. Existem alguns fatores de risco identificáveis para a ocorrência de nefropatia associada ao contraste, como depleção do volume arterial efetivo circulante, diabetes melito, mieloma múltiplo, utilização de outras drogas nefrotóxicas ou de volu- mes de contraste $>3-5 \mathrm{ml} / \mathrm{kg}$. Existe evidência do benefício da utilização de contrastes de baixa osmolaridade não iônicos na prevenção da nefropatia por contraste em pacientes de maior risco, assim como evidência preliminar favorável à utilização de contrastes não-iônicos isosmolares nesta prevenção. A utilização profilática de $\mathrm{N}$-acetilcisteína ainda é controversa na literatura, sendo seu benefício mais provável em pacientes com utilização de menores volumes de contraste. Medida de prevenção comprovadamente eficazé a pré e pós-hidratação com solução salina $0,45 \%(1 \mathrm{ml} / \mathrm{kg} / \mathrm{h} 12 \mathrm{hs}$. antes e após o procedimento). A utilização profilática de dopamina, fenoldopan, furosemida, manitol e bloqueadores de canais de cálcio não se mostrou eficaz até o momento; $\boldsymbol{d}$ ) reações alérgicas: reações anafilactóides graves ocorrem em $0,1 \%$. Os contrastes de baixa osmolaridade, em alguns trabalhos, mostraram uma redução na ocorrência de reações alérgicas; $\boldsymbol{e})$ efeitos trombóticos: os contrastes iônicos possuem efeitos anticoagulantes e antiplaquetários confirmados in vitro e in vivo. Existe controvérsia na literatura, mas há um aparente aumento na incidência de complicações trombóticas em pacientes com síndromes coronarianas agudas submetidos à intervenção percutânea com a utilização de contrastes de baixa osmolaridade não iônicos. Não se sabe o potencial deste potencial efeito trombogênico em pacientes que recebem esquemas antiplaquetários mais agressivos, incluindo os inibidores da GpIIb/IIIa.

5.4 - Custo - Os contrastes de baixa osmolaridade custam significativamente mais do que os de alta osmolaridade. Estima-se que o custo adicional nos EUA da utilização rotineira de contrastes de baixa osmolaridade-não iônicos seria de US\$ 1,1 bilhão.

5.5 - Escolha do contraste - Os contrastes de baixa osmolaridade apresentam uma série de benefícios em relação aos de alta osmolaridade. Apresentam menor incidência de efeitos eletrofisiológicos e hemodinâmicos adversos, reações alérgicas, sobrecarga volêmica e nefropatia por contraste, sendo, neste último, o benefício atribuído aos de baixa osmolaridade e isosmolares não iônicos. No entanto, a grande limitação de sua utilização rotineira é o custo. Existe controvérsia na literatura em relação à escolha do contraste, mas a maioria dos especialistas recomenda uma utilização de contrastes de baixa osmolaridade prudente e custo efetiva, ou seja, apenas em subgrupos de pacientes de maior ris- 
cos para os efeitos adversos descritos. São pacientes considerados de risco os que apresentam instabilidade hemodinâmica ou eletrofisiológica, insuficiência cardíaca descompensada ou pacientes com classe funcional IV (NYHA), insuficiência renal, história de alergia prévia ao contraste, estenose aórtica grave, lesão do tronco da coronária esquerda e síndromes coronarianas agudas. Também é recomendada a utilização de contrastes de baixa osmolaridade na injeção em enxertos envolvendo a artéria torácica interna (mamária) e em arteriografias periféricas, devido ao menor desconforto associado. Apesar da controvérsia existente em relação às complicações trombóticas, recomenda-se a utilização de contrastes de baixa osmolaridade-iônicos em pacientes com síndromes coronarianas agudas.

\section{Admissão e alta do paciente}

A permanente evolução das técnicas e materiais para a realização de procedimentos invasivos em cardiologia temnos permitido realizá-los de maneira mais simples e segura. Este fato, aliado a uma crescente demanda pela redução de custos, tem levado, cada vez mais, a uma internação hospitalar convencional não freqüente para a realização de procedimentos diagnósticos em cardiologia intervencionista.

Para a maioria dos casos, tem sido suficiente a observação do paciente em caráter de hospital - dia, com período médio de observação de 8 horas após o término do procedimento.

Constituem exceção a esta orientação, de maneira geral 3 situações: 1) ocorrência de complicações no procedimento (ex: formação de grande hematoma); 2) achados revelados pelo procedimento que requerem hospitalização (ex: grave obstrução no tronco da coronária esquerda); 3 ) necessidade de cuidados especiais por causas não cardíacas (ex: insuficiência renal crônica).

Para os pacientes que serão submetidos à intervenção terapêutica, recomenda-se a internação hospitalar por $24 \mathrm{~h}$, ainda que, em alguns centros internacionais, sejam realizados procedimentos com alta no mesmo dia. Merece destaque a infreqüente necessidade de encaminhar o paciente para o CTI e mesmo para a Unidade Coronariana. Cumpre ainda ressaltar a necessidade de o paciente fornecer Consentimento Informado para a realização do procedimento, com clara menção de que lhe foi dado conhecimento da freqüência de ocorrência das possíveis complicações inerentes ao procedimento.

\section{Preparo de pacientes em situações especiais}

As recomendações a seguir são baseadas na opinião de especialistas e nem sempre possuem comprovação científica com evidência estatística.

7.1 - Pacientes em uso de anticoagulante oral ou heparina - Interromper o uso do cumarínico por 2-4 dias até RNI $<1,5-1,8$. Dependendo da condição clínica (alto risco de ocorrência ou recorrência de fenômeno tromboembólico), o paciente deverá ser heparinizado (heparina de baixo peso molecular ou não fracionada, em regime de internação ou não). Caso o procedimento seja de urgência e o rni > 1,5, deverá ser feita reserva de plasma fresco congelado e utilização de Vit. K parenteral em doses baixas para a reversão do efeito do cumarínico, lembrando que a administração de Vit. K poderá tornar o paciente refratário à anticoagulação terapêutica por dias. Dependendo da condição clínica, o paciente deverá ser heparinizado, assim que possível, após o procedimento. Pacientes heparinizados podem realizar procedimentos angiográficos com segurança, havendo, no entanto, necessidade de hemostasia rigorosa e, às vezes, utilização de protamina no final. Para pacientes em uso de heparina não fracionada, idealmente deve-se realizar o TCA, para avaliar a necessidade de administração de suas doses adicionais, no caso de intervenções percutâneas. No caso de pacientes em uso terapêutico de heparinas de baixo peso molecular em que a última dose foi administrada em período inferior a $8 \mathrm{~h}$ da realização do procedimento, não há necessidade de dose adicional de heparina.

7.2 - Pacientes em uso de agentes antiplaquetários - Independente da via de administração, de sua associação ou não, e da via de acesso do procedimento, os agentes antiplaquetários não necessitam ser interrompidos previamente.

7.3 - Pacientes em uso de sildenafil (Viagra) - Interromper o uso da medicação 24 hs antes do procedimento. Em caso de procedimentos de urgência, interromper o uso da medicação e somente utilizar nitratos (qualquer formulação) em último caso. Episódios de grave hipotensão podem ocorrer em pacientes em uso desta medicação e devem ser tratados adequadamente (Infusão de volume, vasopressores e BIA, caso necessário.

7.4 - Pacientes em uso de metformim - Pacientes em uso de metformim exibem um risco aumentado de desenvolvimento de acidose láctica após a administração de contrastes iodados. Essa complicação é extremamente rara, porém com uma mortalidade em torno de $50 \%$.

Em pacientes com função renal normal ou desconhecida, a medicação, idealmente, deve ser suspensa $24-48 \mathrm{~h}$ antes do procedimento, e reiniciada caso o paciente e a creatinina sérica estejam estáveis após 48h. Não há necessidade de suspensão do procedimento, caso não tenha sido interrompida a medicação nas 24-48 h anteriores ao mesmo, no entanto os cuidados e a vigilância no per e pós- procedimento devem ser intensificados. Recomenda-se hidratação adequada antes a após o procedimento.

Em pacientes com disfunção renal $(\mathrm{Cr}>1,5 \mathrm{mg} / \mathrm{dl})$, a medicação deve ser suspensa 24-48h antes do procedimento e somente reiniciada após confirmação da estabilidade da creatinina sérica e do quadro clínico do paciente. Recomenda-se suspensão de procedimentos eletivos em caso de não suspensão prévia da medicação Caso o procedimento seja de urgência/emergência, os cuidados per e pós-procedimento devem ser intensificados, recomendando-se o uso de contrastes de baixa osmolaridade-não iônicos e hidratação adequada.

7.5 - Pacientes com história de alergia a contraste Devido à ocorrência rara de reações alérgicas verdadeiras aos contrastes é difícil a recomendação de um preparo ade- 
quado com segurança. Os protocolos de pré-medicação variam e nenhum é comprovadamente superior ao outro. $\mathrm{O}$ protocolo mais recomendado é o seguinte: prednisona 20 mg - 2 cps Vo 6/6 h, iniciadas 12-18h antes do procedimento; difenidramina $50 \mathrm{mg}$ - Vo ou Iv antes do procedimento; utilização de contrastes de baixa osmolaridade

Em casos de procedimentos de urgência/emergência, recomenda-se a administração de $40 \mathrm{mg}$ Ev de metilprednisolona ou equivalente

Na maioria dos serviços, entretanto, esse protocolo não é seguido, sendo apenas feita a administração EV de corticosteróides horas antes do procedimento, não havendo dados que recomendem ou contra-indiquem a prática.

7.6 - Pacientes com risco de nefropatia induzida pelo contraste - Pacientes com função renal normal podem desenvolver insuficiência renal aguda após a administração de contrastes iodados, sendo fatores de risco identificáveis: hipovolemia, uso concomitante de drogas nefrotóxicas, utilização de volumes de contraste $>3 \mathrm{ml} / \mathrm{kg}$, diabetes melito e mieloma múltiplo. Deve-se estar atento para a correção, caso seja possível, desses fatores de risco.

Em pacientes com disfunção renal em níveis não dialíticos(cr > 1,5 mg/dl), especialmente os com nefropatia diabética, pode haver piora significativa da função renal, requerendo-se maior atenção em relação aos fatores de risco conhecidos e a aplicação de outras medidas preventivas, sendo as recomendadas: utilização de contrastes de baixa osmolaridade não iônicos ou isosmolares (com limitação do volume de contraste, por vezes com a não realização da ventriculografia e com o adiamento de intervenções percutâneas eletivas após exames diagnósticos); hidratação antes e após o procedimento com solução salina $100-150 \mathrm{ml} / \mathrm{h}$ por 8-12hs, desde que a condição clínica do paciente permita essa sobrecarga volêmica; pré-medicação com N-acetilcistéina (600 mg Bid por 2 dias, iniciados no dia anterior ao procedimento); acompanhamento clínico e dos níveis séricos de creatinina.

Pacientes com insuficiência renal crônica, já em programas de diálise, devem ter avaliação concomitante do nefrologista na decisão da conduta, devendo-se, no entanto, limitar o volume de contraste e utilizar contrastes de baixa osmolaridade

7.7 - Pacientes diabéticos- insulino requerentes - Os pacientes devem receber metade a $1 / 3$ da dose de insulina pela manhã, mantidos em jejum e receber infusão venosa contendo glicose. A posterior liberação da dieta segue a rotina de pacientes não diabéticos.

Idealmente, os procedimentos nesses pacientes devem ser realizados na parte da manhã, a fim de evitar longos períodos de jejum após a administração da insulina.

\section{Referências Consultadas}

1. American College of Cardiology/Society for Cardiac Angiography and Interventions- Clinical Expert Consensus Document on Cardiac Catheterization Laboratory Standarts J Am Coll Cardiol 2001; 37: 2170-214

2. Guidelines for Coronary Angiography - J Am Coll Cardiol 1999; 33: 1756-824

3. Use of Nonionic or low osmolar contrast agents in cardiovascular procedures. J Am Coll Cardiol 1993; 21: 269-73
4. Donald S. Baim, William Grossman. Grossman's Cardiac Catheterization, Angiography and Intervention $-2000-6^{\text {th }}$ edition

5. Morton J. Kern. The Cardiac Catheterization Handbook $1999 ; 3^{\text {rd }}$ edition

6. Robert Safian, Mark Freed. The Manual of Interventional Cardiology $2002 ; 3^{\text {rd }}$ edition.

7. Peter Aspelin et al. Nephotoxic effects in high-risk patients undergoing angiography. N Engl J Med 2003; 348: 491-9. 